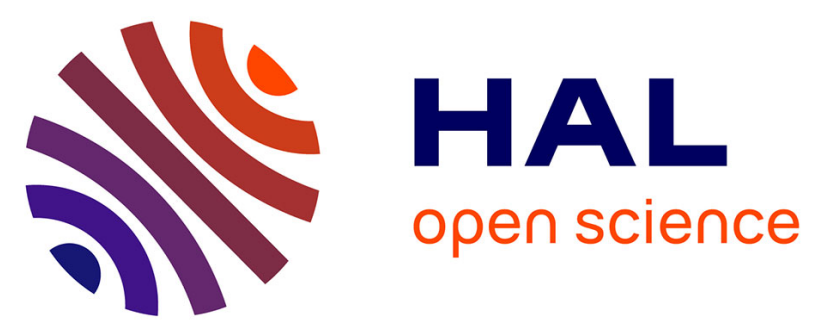

\title{
Low baseline and subsequent higher aortic abdominal aneurysm FDG uptake are associated with poor sac shrinkage post endovascular repair
}

Pierre-Yves Marie, Didier Plissonnier, Stéphanie Bravetti, Raphael Coscas, Martin Rouer, Stéphan Haulon, Damien Mandry, Jean-Marc Alsac, Sergueï

Malikov, Nicla Settembre, et al.

\section{To cite this version:}

Pierre-Yves Marie, Didier Plissonnier, Stéphanie Bravetti, Raphael Coscas, Martin Rouer, et al.. Low baseline and subsequent higher aortic abdominal aneurysm FDG uptake are associated with poor sac shrinkage post endovascular repair. European Journal of Nuclear Medicine and Molecular Imaging, 2018, 45 (4), pp.549-557. 10.1007/s00259-017-3883-1 . hal-03254749

\section{HAL Id: hal-03254749 \\ https://hal.univ-lorraine.fr/hal-03254749}

Submitted on 16 Jul 2021

HAL is a multi-disciplinary open access archive for the deposit and dissemination of scientific research documents, whether they are published or not. The documents may come from teaching and research institutions in France or abroad, or from public or private research centers.
L'archive ouverte pluridisciplinaire HAL, est destinée au dépôt et à la diffusion de documents scientifiques de niveau recherche, publiés ou non, émanant des établissements d'enseignement et de recherche français ou étrangers, des laboratoires publics ou privés. 


\title{
Low baseline and subsequent higher aortic abdominal aneurysm FDG uptake are associated with poor sac shrinkage post endovascular repair
}

\author{
Pierre-Yves Marie ${ }^{1,2,3} \cdot$ Didier Plissonnier $^{4} \cdot$ Stéphanie Bravetti $^{5} \cdot$ Raphael Coscas $^{6} \cdot$ Martin Rouer $^{4} \cdot$ Stéphan Haulon $^{7}$. \\ Damien Mandry ${ }^{5,8}$. Jean-Marc Alsac ${ }^{9} \cdot$ Sergueï Malikov $^{10} \cdot$ Nicla Settembre $^{10} \cdot$ Yann Gouëffic $^{11} \cdot$ Olivier Morel $^{12}$. \\ Véronique Roch ${ }^{1} \cdot$ Emilien Micard $^{8,13} \cdot$ Zohra Lamiral $^{13} \cdot$ Jean-Baptiste Michel ${ }^{14}$ - Patrick Rossignol ${ }^{2,13}$
}

\begin{abstract}
Purpose The growth phases of medically treated abdominal aortic aneurysms (AAA) are frequently associated with an ${ }_{18} \mathrm{~F}-$ fluorodesoxyglucose positron emission tomography (FDG-PET) pattern involving low baseline and subsequent higher FDG uptake. However, the FDG-PET patterns associated with the endovascular aneurysm repair (EVAR) of larger AAA are presently unknown. This study aimed to investigate the relationship between serial AAA FDG uptake measurements, obtained before EVAR and 1 and 6 months post-intervention and subsequent sac shrinkage at 6 months, a well-recognized indicator of successful repair. Methods Thirty-three AAA patients referred for EVAR (maximal diameter: $55.4 \pm 6.0 \mathrm{~mm}$, total volume: $205.7 \pm 63.0 \mathrm{~mL}$ ) underwent FDG-PET/computed tomography (CT) before EVAR and at 1 and 6 months thereafter, with the monitoring of AAA volume and of a maximal standardized FDG uptake [SUVmax] averaged between the axial slices encompassing the AAA. Results Sac shrinkage was highly variable and could be stratified into three terciles: a first tercile in which shrinkage was absent or very limited $(0-29 \mathrm{~mL})$ and a third tercile with pronounced shrinkage $(56-165 \mathrm{~mL})$. SUVmax values were relatively low at baseline in the 1st tercile (SUVmax: $1.69 \pm 0.33)$, but markedly increased at 6 months $(2.42 \pm 0.69, p=0.02$ vs. baseline). These SUV max values were by contrast much higher at baseline in the 3rd tercile (SUVmax: $2.53 \pm 0.83 p=0.009$ vs. 1st tercile) and stable at 6 months $(2.49 \pm 0.80)$, while intermediate results were documented in the 2nd tercile. Lastly, the amount of sac shrinkage, expressed in absolute values or in percentages of baseline AAA volumes, was positively correlated with baseline SUVmax ( $p=0.001$ for both).

Conclusion A low pre-EVAR FDG uptake and increased AAA FDG uptake at 6 months are associated with reduced sac shrinkage. This sequential FDG-PET pattern is similar to that already shown to accompany growth phases of medically treated AAA.
\end{abstract}

Raphael Coscas and Martin Rouer contributed equally to this work.

Pierre-Yves Marie

py.marie@chru-nancy.fr

1 CHRU-Nancy, Université de Lorraine, Nuclear Medecine \& Nancyclotep Platform, Nancy F-54000, France

2 INSERM, University of Lorraine, UMR 1116, F-54000 Nancy, France

3 CHRU-Nancy, Hôpitaux de BRABOIS, Service de Médecine Nucléaire, Allée du Morvan, 54500 Vandœuvre, France

4 Department of Vascular Surgery, CHU-Rouen, F-76000 Rouen, France

5 CHRU-Nancy, Université de Lorraine, Department of Radiology, Nancy F-54000, France

6 Chirurgie Vasculaire, Hôpital Ambroise Paré, APHP, F-92100 Boulogne-Billancourt, France

7 Department of Vascular Surgery, CHU-Lille, F-59000 Lille, France

8 INSERM, University of Lorraine, UMR 947, F-54000 Nancy, France

9 Department of Vascular Surgery, APHP, HEGP, F-75000 Paris, France

10 CHRU-Nancy, Université de Lorraine, Vascular Surgery, Nancy F-54000, France

11 Department of Vascular Surgery, CHU-Nantes, F-44000 Nantes, France

12 Department of Nuclear Medecine, CHU-Besançon, F-25000 Besançon, France

13 INSERM, CHRU-Nancy, Université de Lorraine, CIC-1433, FCRIN INI-CRCT, F-54000 Nancy, France

14 INSERM, Bichat, UMR 698, F-75000 Paris, France 
Keywords Abdominal aortic aneurysms $\cdot$ Endovascular aneurysm repair $\cdot$ CT angiography $\cdot$ Positron emission tomography

${ }^{18} \mathrm{~F}$-fluorodesoxyglucose

\section{Introduction}

A growing number of patients with an abdominal aortic aneurysm (AAA) are treated by endovascular aneurysm repair (EVAR) in order to exclude the AAA sac from blood flow and pressure $[1,2]$. EVAR was recently shown to confer an early survival benefit although with a conversely lower late survival compared with open surgical repair [3]. A significant shrinkage of the AAA volume after EVAR is a proof of successful repair, yielding evidence of a low risk of late complications $[1,2,4-6]$. This shrinkage is a progressive phenomenon, with a post-intervention delay time of at least 6 months currently recommended for its assessment $[1,2,4,7,8]$. However, the degree of shrinkage is highly variable and difficult to predict, although has been found related to initial maximal diameter and thrombus volume and to the presence of endoleak and endograft type [7, 9-13].

In the present study, ${ }^{18} \mathrm{~F}$-fluorodesoxyglucose positron emission tomography (FDG-PET) was used to characterize the glycolytic metabolism of the AAA walls after EVAR with the monitoring of maximum standardized uptake values (SUVmax) averaged between the axial slices encompassing the AAA and in keeping with previous studies where this SUVmax value was found to be associated with the growth phases of the asymptomatic and uncomplicated forms of medically-treated AAA [14-16]. These growth phases were indeed shown to be 1) preceded by a phase with a low baseline SUVmax [14, 15], but also 2) accompanied by a subsequent increase in this SUVmax value [14].

However, it is not known whether this or other patterns may be documented in relation to patient outcome in larger AAA, which are increasingly treated by EVAR. The present prospective multicenter study was thus primarily aimed at investigating the relationship between serial AAA FDG uptake measurements, obtained before EVAR and 1 and 6 months post-intervention, and subsequent sac shrinkage at 6 months, a well-recognized indicator of successful repair.

\section{Materials and methods}

\section{Patient selection and study design}

The study was prospectively conducted in five French University Hospitals (CHU): Lille, Nancy, Nantes, Rouen and the Georges Pompidou European Hospital (HEGP) in Paris. The protocol was approved by an Ethics Committee (CPP agreement $\left.n^{\circ} 07.11 .03\right)$ and released on the ClinicalTrials.gov site under the identifier NCT01253252.
All study patients gave signed informed consent to participate. The main inclusion criteria were: infra-renal AAA of a common atherosclerotic origin for which an endovascular aneurysm repair (EVAR) was indicated; age $>18$ years; no contraindication for performing FDG-PET or CT angiography; and no known disease leading to liver dysfunction or to marked inflammatory response (cancer, autoimmune disease, tuberculosis, Crohn's disease, etc.).

The study patients were referred for the separate recordings of FDG-PET and CT angiography at three time-points: prior to EVAR and at approximately 1 month (from 20 to 30 days) and 6 months ( \pm 10 days) thereafter.

\section{Hybrid PET/CT recording}

FDG-PET images were recorded in each of the five centers with hybrid systems involving a multi-detector CT: Philips Gemini GXL 16 in Paris, Siemens Biograph TruePoint in Nancy, Biograph Sensation $16 \mathrm{Hi}-\mathrm{Rez}$ in Rouen, Siemens Biograph mCT 40 in Nantes and General Electric Discovery $\mathrm{RX}$ in Lille. A low dose FDG-PET/CT protocol, with a recording focused on the AAA area, was implemented. An activity of only $2.75 \mathrm{MBq}$ per $\mathrm{Kg}$ of body weight, with a minimum of $220 \mathrm{MBq}$, was injected intravenously after an overnight fast. The blood glucose level was previously verified to ensure a level of $<1.5 \mathrm{~g} \mathrm{~L}^{-1}$ in all patients. Imaging was initiated 90 min later, as recommended for decreasing the blood signal on vascular FDG-PET images [17], starting with the CT recording of the abdomen ( 110 to $130 \mathrm{kV}$, intensity adapted to noise index, $512 \times 512$ matrix, 3 to $5 \mathrm{~mm}$ slice thickness) and followed by a 3D PET recording, involving two to three bed positions of $12 \mathrm{~min}$ each, all patients being scanned from $5 \mathrm{~cm}$ above the renal bifurcation to $10 \mathrm{~cm}$ below the iliac bifurcation.

The CT and PET images were recorded and reconstructed with the parameters recommended for clinical routine for each system, but with the constrain of using cubic voxels of 3 to $4 \mathrm{~mm}$ for PET. PET images were corrected for scatter and attenuation, and standardized uptake values (SUV) were calculated by dividing the activity measured in each voxel by the total injected activity expressed per gram of body weight and corrected for radioactive decay.

\section{Recording of CT angiography}

Angiographic images of AAA were recorded with various CT scanners: General Electric LightSpeed VCT in Nancy, Paris, Lille and Nantes, a General Electric Discovery CT750 HD or a Siemens Sensation 16 in Rouen and a hybrid PET/CT 
General Electric Discovery RX in Lille. A volume of 1.5 to $2 \mathrm{~mL} / \mathrm{kg}$ of iodinated contrast, at an iodine concentration of 300 to $400 \mathrm{mg} \cdot \mathrm{mL}^{-1}$, was injected in an upper limb vein at a rate of 3 to $5 \mathrm{~mL} / \mathrm{s}$, and was followed by 20 to $60 \mathrm{~mL}$ of saline solution. The CT images were reconstructed using a $512 \times 512$ matrix and a 1 to $1.25 \mathrm{~mm}$ slice thickness.

\section{Analysis of hybrid PET/CT images}

The FDG-PET data were collected by a single experienced observer with a paired display of CT and fused PET/CT images $[14,18,19]$ and using a dedicated software (Venus Explorer 142, Nicesoft, Paris, France). As previously described [14] and illustrated in Fig. 1, maximum standardized uptake values (SUVmax) were collected on consecutive transaxial slices encompassing the abdominal aorta, with a manual drawing of regions of interest (ROI) around the aorta. These SUVmax values were averaged between slices covering the AAA volume, with the exclusion of the inferior and superior extremities (at a distance of $1 \mathrm{~cm}$ from each neck region). In a previous sub-group of 18 patients having undergone the same PET/CT procedure for a medically treated AAA [14], the relative variations in these average SUVmax values between two consecutive PET recordings was $+1.5 \pm$ $17.9 \%$.

Fig. 1 Left panel: representation of the approach used to determine the mean SUVmax value for each abdominal aortic aneurysm (AAA) and at each time-point on the fused PET/CT images while averaging SUVmax values from the axial slices encompassing the AAA [14]; right panel: 3Drendering views of the external limits of the total AAA volume obtained from CT-angiography after segmentation with the dedicated software [20,21], the distribution of thrombus thickness being displayed through a color scale

\section{Analysis of CT angiography}

The maximal diameter and the total volume of the AAA, as well as the volumes of the luminal and thrombus portions of the AAA, were assessed by a single experienced observer (SB) using the A3Dmax segmentation software (Object Research System, Montreal, Canada) [20, 21]. This software provides a robust segmentation of the lumen and thrombus volumes of the AAA [20] even after EVAR treatment [21]. The upper and lower limit planes were systematically placed at the levels of the inferior renal artery and the aorto-iliac bifurcation, respectively, and the amount of shrinkage was defined as the absolute decrease in total volume of the AAA between baseline and the 6-month control, a delay time considered as sufficient for this purpose $[1,2,4,7,8]$. The presence and grading of endoleaks was obtained through a consensual visual analysis provided by two experienced observers (RC, MR).

\section{Statistical analysis}

Continuous variables are expressed as means \pm SD and categorical variables as percentages. Paired comparisons between baseline and the 1- or 6-month controls were performed using Wilcoxon rank sum tests for continuous variables and
PET/CT fusion images

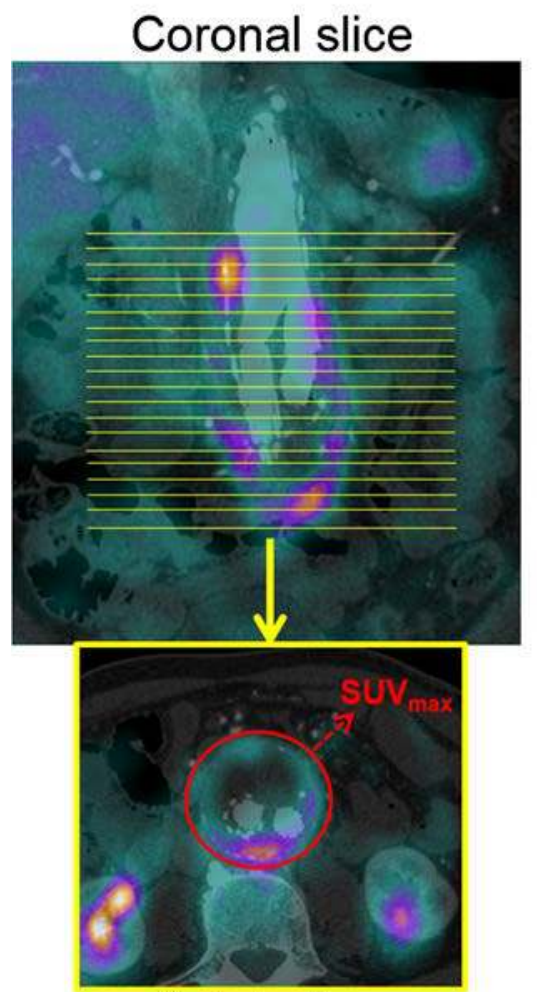

Axial slice

\section{CT angiography}

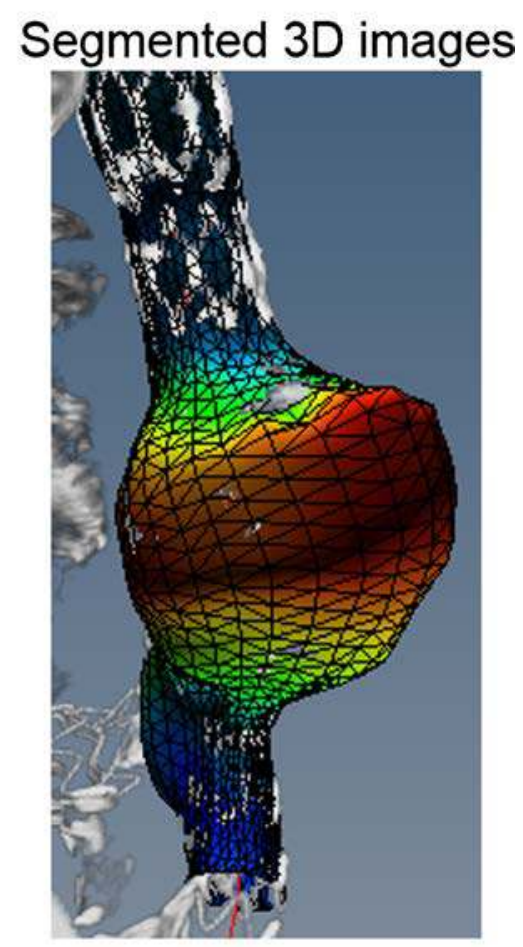


McNemar tests for discrete variables (Table 1). Relationships between baseline imaging parameters and amount of sac shrinkage at 6 months (in $\mathrm{mL}$ ) were assessed using Spearman's rank correlation coefficients for continuous variables (Table 2).

ANOVA models were additionally used for assessing the relationships between the amount of shrinkage, a fixed factor expressed into terciles, and baseline $\mathrm{CT}$ parameters or repeated SUVmax measurements (baseline, 1-month, 6-month) as dependent variables (Table 3 ).

In addition, multivariable linear regression analyses were performed to assess the relationships between amount of sac shrinkage, as dependent variable, and the following independent baseline parameters: AAA volume, SUVmax, age, body mass index, tobacco use, blood glucose, blood pressure, EVAR system, $\mathrm{C}$ reactive protein and medications (statins, angiotensin converting enzyme inhibitors, angiotensin receptor blockers, antithrombotic agents, antidiabetics and betablockers). Among these factors, those significantly associated with the amount of sac shrinkage with a $p$-value $<0.15$ at univariate regression were further selected using an interactive backward selection process. Only the factors associated with the amount of sac shrinkage with a $p$-value $<0.10$ were retained in the final models.

Validity of the model assumptions was verified (assumption of normality of residuals, test for heteroscedasticity and multicollinearity by examining the variance inflation factors). A two-sided $p$ value less than 5\% was considered significant except for the multivariate models where this threshold was set to $10 \%$ owing to the limited number of observations and to the exploratory nature of the study. Bonferroni correction was performed to control the effect of multiple testing, and the $p$ values of post hoc pairwise comparisons were adjusted.

\section{Results}

A total of 43 patients were initially included although 10 had to be subsequently excluded: eight due to the lack of FDGPET exam at baseline or at 6 months, one due to a technical recording problem of FDG-PET images and one with an unachievable delineation of the AAA volume (contiguous large iliac aneurysm). Thus, 33 patients constituted the final study population. Eighteen were investigated in Rouen, five in Lille, four in Nancy, four in Paris and two in Nantes. Only non-injected CT images were available for 8 exams due to technical issues and/or the advent of a contraindication to contrast injection during follow-up (evolving kidney disease). Total AAA volumes could be monitored in all 33 patients, while lumen and thrombus volumes could be obtained from the $\mathrm{CT}$ angiographies of 30 patients at baseline, 32 at 1 month and 29 at 6 months.

\section{Baseline data}

The mean age of the 33 patients was $70 \pm 7$ years. All were men and $11(33.3 \%)$ had a previous history of cardiovascular disease (coronary artery disease nine 9, peripheral arterial disease in two). All but three (91\%) had a history of smoking, 19 $(58 \%)$ were treated for hypertension, six $(18 \%)$ were treated for diabetes and $19(58 \%)$ were treated by statins.

The main baseline imaging data are detailed in Table 1. At $\mathrm{CT}$ angiography, the maximal diameter of the AAA was 55.4 $\pm 6.0 \mathrm{~mm}$ on average (from 45.6 to $73.3 \mathrm{~mm}$ ), and the mean total volume of the AAA was $205.7 \pm 63.0 \mathrm{~mL}$. Mean SUVmax at baseline FDG-PET was $2.23 \pm 0.68$.

EVAR was performed with a mean delay time of $14 \pm 13$ days after baseline FDG-PET using six different systems: 27 Zenith (Cook Inc., Bloomington, IN, USA), two Endurant (Medtronic Inc., Minneapolis, MN, USA), one Talent (Medtronic Inc., Minneapolis, MN, USA), one Excluder (Gore Inc., Flagstaff, AZ, USA), one Anaconda (Vascutek Terumo Inc., Ichinnan, Scotland, UK.) and one AFX (Endologix, Irvine, CA, USA).

\section{Follow-up}

As detailed in Table 1, there were significant decreases between baseline and the 1- and 6-month time-points for both total AAA volumes and AAA lumen volumes. However, significant decreases were documented only at 6 months for
Table 1 Paired comparisons of the main PET/CT parameters between baseline and 1- and 6month controls

\begin{tabular}{llll}
\hline & Baseline & 1 month & 6 months \\
\hline Maximal diameter $(\mathrm{mm})$ & $55.4 \pm 6.0$ & $56.0 \pm 7.9$ & $52.8 \pm 6.7 *$ \\
Total AAA volume $\left(\mathrm{cm}^{3}\right)$ & $205.7 \pm 63.0$ & $168.7 \pm 65.3 *$ & $160.0 \pm 56.4 *$ \\
$\quad$ lumen volume $\left(\mathrm{cm}^{3}\right)$ & $71.3 \pm 25.4$ & $30.6 \pm 12.0 *$ & $35.3 \pm 15.6 *$ \\
$\quad$ - thrombus volume $\left(\mathrm{cm}^{3}\right)$. & $137.9 \pm 40.7$ & $139.7 \pm 57.5$ & $128.3 \pm 43.9$ \\
Type 2 endoleak & & $14(42 \%)$ & $13(39 \%)$ \\
SUVmax & $2.23 \pm 0.68$ & $2.32 \pm 0.68$ & $2.59 \pm 0.67 *$ \\
\hline
\end{tabular}

$A A A$ abdominal aortic aneurysm, $B P$ blood pressure, $C T$ X-ray computed tomography, $F D G-P E T^{18} \mathrm{~F}-$ fluorodesoxyglucose positron emission tomography, SUVmax maximal standardized uptake value ${ }^{*} p<0.05$ for paired comparison vs. baseline with Bonferroni adjustment for multiple testing 
Table 2 Spearman correlation coefficients (and associated $p$ values) for relationships between the amount of sac shrinkage at 6 months (in $\mathrm{mL}$ ) and selected imaging parameters

\begin{tabular}{ll}
\hline Maximal diameter & $+0.12(0.53)$ \\
\hline Total AAA volume $\left(\mathrm{cm}^{3}\right)$ & $+0.47(0.006) *$ \\
- lumen volume $\left(\mathrm{cm}^{3}\right)$ & $+0.61(<0.001) *$ \\
- thrombus volume $\left(\mathrm{cm}^{3}\right)$ & $+0.41(0.03) *$ \\
SUVmax & \\
at baseline & $+0.55(0.001) *$ \\
at 1 month & $+0.03(0.86)$ \\
at 6 months & $+0.05(0.76)$ \\
6 months minus baseline & $-0.51(0.002) *$ \\
\hline
\end{tabular}

$A A A$ abdominal aortic aneurysm, $C T \mathrm{X}$-ray computed tomography, $F D G$ $P E T{ }^{18} \mathrm{~F}$-fluorodesoxyglucose positron emission tomography, SUVmax maximal standardized uptake value

$* p<0.05$ for Spearman correlation

maximal AAA diameter with no significant change noted at either 1 or 6 months for thrombus volume. The 6-month decrease in total AAA volume, reflecting the absolute amount of sac shrinkage, was highly variable and could be stratified into three terciles, the first in which shrinkage was absent or very limited $(0-29 \mathrm{~mL})$ and a third where shrinkage was pronounced $(56-165 \mathrm{~mL})$. Only type- 2 endoleaks were documented, with rates of $42 \%$ at 1 month and $39 \%$ at 6 months.

Significant increases in SUVmax were noted at 6 months $(2.23 \pm 0.68$ vs. $2.59 \pm 0.67, p=0.004)$.

\section{Correlates of sac shrinkage}

The absolute amount of sac shrinkage at 6 months was not significantly associated with clinical data such as active smoking, statin treatment, diabetes treatment and presence of endoleak.

By contrast, this absolute amount of sac shrinkage at 6 months was positively correlated with the baseline levels of all volumetric AAA parameters (total, lumen and thrombus volumes), as well as to the baseline SUVmax values (Table 2). The amount of shrinkage was also linked to the changes in SUVmax from baseline to 1 or 6 months, i.e. with the higher increases in SUVmax at 1 or 6 months being associated with the lesser amount of shrinkage.

Further analyses, detailed in Table 3 and in Fig. 2, were performed on the population stratified into three terciles according to the amount of sac shrinkage. Patients from the 1st tercile (none or limited shrinkage) exhibited a relatively low AAA volume at baseline $(178.2 \pm 57.8 \mathrm{~mL})$. Their SUVmax values were also rather low at baseline $(1.69 \pm 0.33)$ while increased at 6 months $(2.42 \pm 0.69$, $p=0.02$ for paired comparison vs. baseline). As shown in Fig. 2, this increase in SUVmax had the particularity of being documented as early as at 1 month together with an increase in thrombus volume.

The 3rd tercile patients (marked shrinkage) exhibited higher baseline values for both AAA volume (253.6 \pm $67.7 \mathrm{~mL}, p=0.0154$ vs. 1 st tercile) and SUVmax (2.53 \pm $0.83, p=0.009$ vs. 1 st tercile), with no change in SUVmax or in thrombus volume at 1 or 6 months (SUVmax at 6 months: $2.49 \pm 0.80$ ).

Finally, the 2nd tercile patients (moderate shrinkage) exhibited intermediate results with regard to baseline levels of AAA volume $(190.1 \pm 43.1 \mathrm{~mL})$ and SUVmax $(2.41 \pm 0.33$ at baseline and $2.80 \pm 0.53$ at 6 months).

An example of PET/CT images of a patient from the 1st tercile is given in Fig. 3 .
Table 3 Relationships between selected imaging parameters and a tercile-based classification of the population according to the amount of sac shrinkage, namely absent or limited in a 1 st tercile (< $29 \mathrm{~mL}$ ), mid-to-moderate in a 2nd tercile ( 29 to $56 \mathrm{~mL}$ ) and pronounced in a 3 rd tercile ( $>$ $56 \mathrm{~mL}$ )

\begin{tabular}{lllll}
\hline & 1st tercile & 2nd tercile & 3rd tercile & Overall $p$ value \\
\hline Maximal diameter $(\mathrm{mm})$ & $55.5 \pm 6.4$ & $53.8 \pm 4.7$ & $57.7 \pm 6.9$ & 0.29 \\
Total AAA volume $\left(\mathrm{cm}^{3}\right)$ & $178.2 \pm 57.8$ & $190.1 \pm 43.1$ & $253.6 \pm 67.7^{*}$ & 0.010 \\
$\quad$ - lumen volume $\left(\mathrm{cm}^{3}\right)$ & $51.6 \pm 27.0$ & $68.1 \pm 15.9$ & $90.9 \pm 20.5^{*}$ & 0.002 \\
$\quad$ - thrombus volume $\left(\mathrm{cm}^{3}\right)$ & $119.7 \pm 29.6$ & $129.4 \pm 26.6$ & $162.6 \pm 52.3$ & 0.049 \\
Type 2 endoleak & & & & \\
at 1 month & $4(40 \%)$ & $6(46 \%)$ & $4(40 \%)$ & 1.00 \\
at 6 months & $4(40 \%)$ & $8(62 \%)$ & $1(10 \%)$ & 0.07 \\
SUVmax & & & & \\
at baseline & $1.69 \pm 0.33$ & $2.41 \pm 0.33^{*}$ & $2.53 \pm 0.83^{*}$ & 0.0053 \\
at 1 month & $2.27 \pm 0.52$ & $2.18 \pm 0.53$ & $2.55 \pm 0.95$ & 0.43 \\
at 6 months & $2.42 \pm 0.69$ & $2.80 \pm 0.53$ & $2.49 \pm 0.80$ & 0.34 \\
6 months minus baseline & $0.73 \pm 0.66$ & $0.39 \pm 0.67$ & $-0.04 \pm 0.45$ & 0.028 \\
\hline
\end{tabular}

$A A A$ abdominal aortic aneurysm, $C T$ X-ray computed tomography, FDG-PET ${ }^{18} \mathrm{~F}$-fluorodesoxyglucose, SUVmax maximal standardized uptake value

${ }^{*} p<0.05$ for comparison vs. 1 st tercile with Bonferroni adjustment for multiple testing 
Fig. 2 Comparison of the 6month evolution of lumen, thrombus and total volumes of the abdominal aortic aneurysms (AAA), as well as their FDG uptake, in the study population stratified into three terciles according to "baseline to 6month" shrinkage, namely absent or limited in the first tercile ( $\leq$ $29 \mathrm{~mL}$ ), mid-to-moderate in the second tercile (> $29 \mathrm{~mL}$ and $<56 \mathrm{~mL}$ ) and pronounced in the third tercile ( $\geq 56 \mathrm{~mL})$. PET: positron emission tomography, SUVmax: maximum standardized uptake value. $* p<0.05$ for paired comparison vs. baseline with Bonferroni adjustment
Lumen Volume $(\mathrm{mL})$

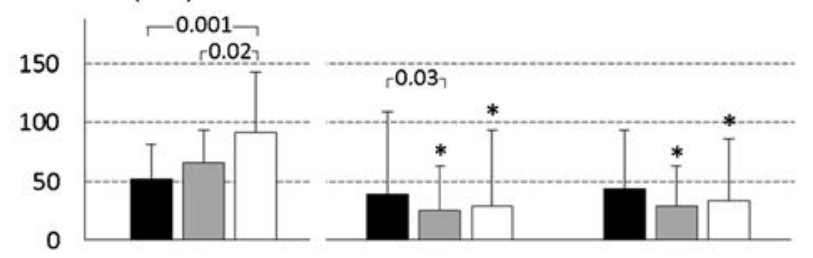

Thrombus Volume $(\mathrm{mL})$

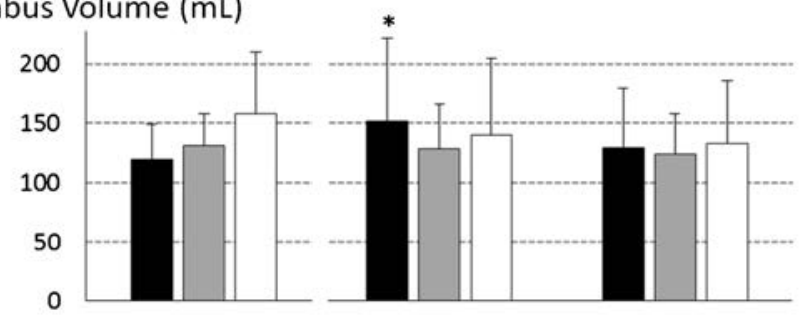

Total Volume $(\mathrm{mL})$

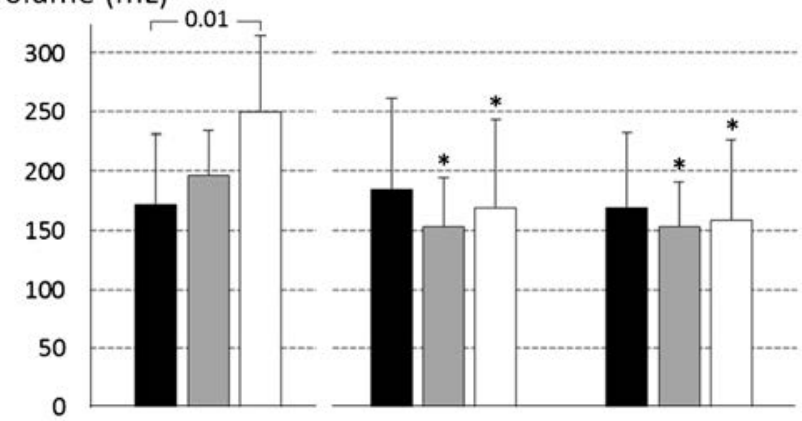

FDG uptake (SUVmax)

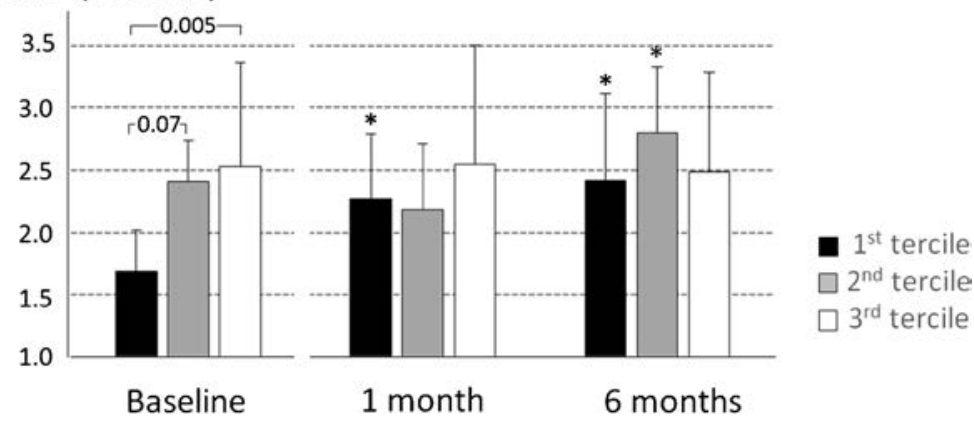

Upon multivariate analysis of the overall study group, the best baseline predictors of the amount of sac shrinkage were: 1) total AAA volume ( $\left.p=0.005, \mathrm{R}^{2}=0.26\right)$ and 2$)$ SUVmax $(p=0.001$, $\mathrm{R}^{2}=0.32$ ). When considering only the 30 patients having undergone a $\mathrm{CT}$ angiography at baseline, the best baseline predictors became: 1) AAA lumen volume $\left(p<0.001, \mathrm{R}^{2}=0.42\right)$ and 2$)$ SUVmax $\left(p=0.08, \mathrm{R}^{2}=0.11\right)$.

The dependence of this absolute amount of shrinkage on baseline levels of both AAA-volume and SUVmax likely explained the strong relationship with baseline SUVmax for the relative amount of shrinkage (i.e., expressed in \% of baseline AAA volume) as detailed in Fig. 4. When the amount of shrinkage was expressed using this \% value, SUVmax was its sole best baseline predictor in the overall study group ( $\left.p=0.001, \mathrm{R}^{2}=0.29\right)$, as well as in the subgroup with CT angiography $\left(p=0.009, \mathrm{R}^{2}=0.22\right)$.

\section{Discussion}

In the present study, low baseline and subsequent higher AAA FDG uptake was found associated with poor sac shrinkage after EVAR. This observation is consistent with the previous findings from medically treated AAAs in which the same sequential FDG-PET pattern was found associated with AAA growth phases [14]. This pattern may thus be considered as a landmark of less favorable outcomes.

In the present study, however, the AAA showing the largest sac shrinkages at 6 months were those showing the highest baseline levels of not only FDG uptake but also of AAA volume (Fig. 2). These two parameters were moreover selected as best independent predictors of the absolute amount of sac shrinkage in our overall population. 


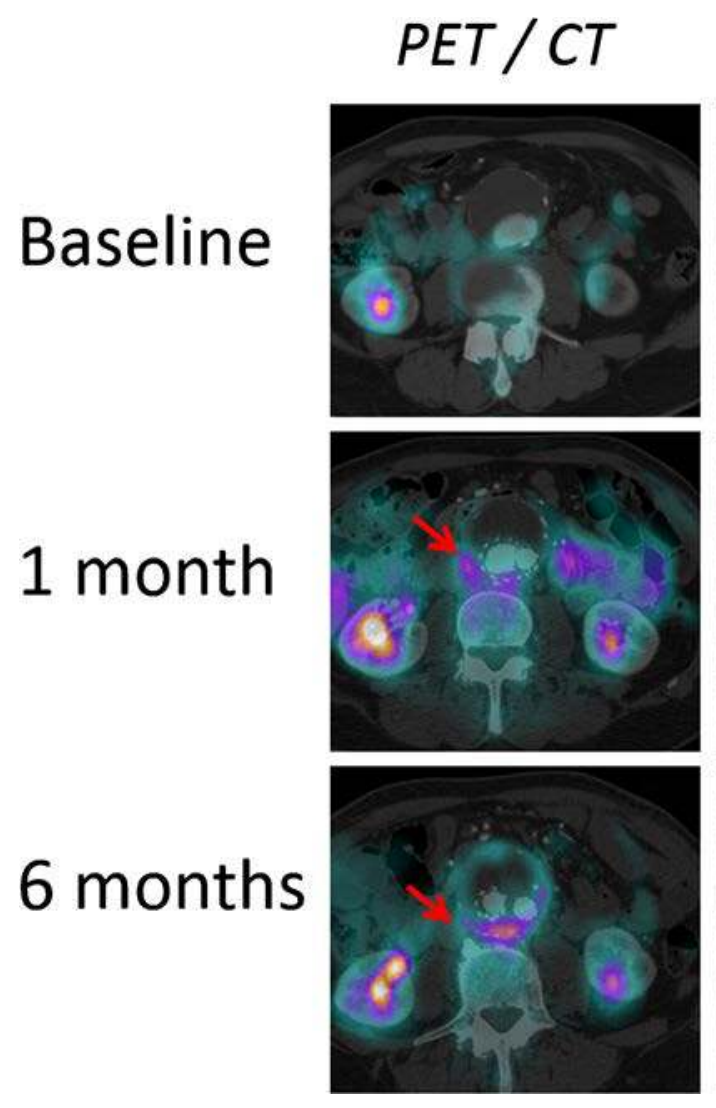

Fig. 3 Representative short-axis slices recorded at the center of the AAA volume with FDG-PET and CT-angiographies performed prior to EVAR (baseline) and at the 1-and 6-month follow-up control points in a patient

Previous studies had already shown that the larger AAA were those most likely to exhibit a significant sac shrinkage after EVAR, even if this observation was mostly obtained through maximal diameter measurements [7]. In the present study, sac shrinkage was defined through volume changes rather than diameter changes, a choice likely to enhance the robustness of the results [22].

It should be noted, however, that the baseline volume of the AAA lumen was selected (as opposed to total AAA volume) when the multivariate prediction of sac shrinkage was performed in patients for whom the lumen and thrombus volumes could be determined (i.e. those with contrast injection during $\mathrm{CT}$ ). This luminal portion may indeed be expected to regress proportionally to its initial volume when the AAA sac is excluded from both blood flow and pressure after EVAR. By contrast, the regression of the thrombus portion of the AAA volume is further contingent on the variable absorption rates of the thrombus [2], rendering this metabolic parameter a possible source of further imprecision for shrinkage prediction.

Nevertheless, in the present study, a low baseline level of FDG uptake was a predictor of limited sac shrinkage, independently of the baseline AAA volumes. This point is best illustrated in Fig. 4 by the inverse relationship between SUVmax and the amount of sac shrinkage expressed in
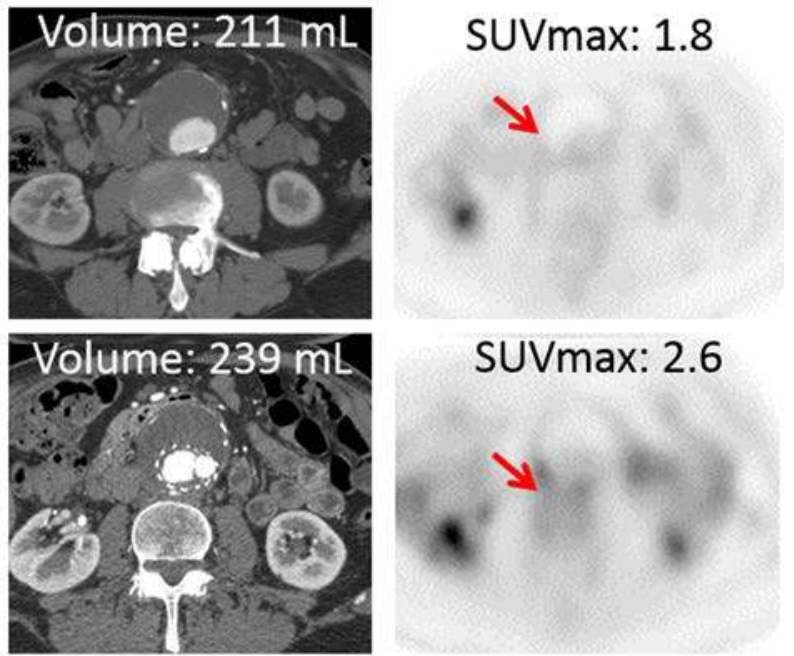

SUVmax: 2.6
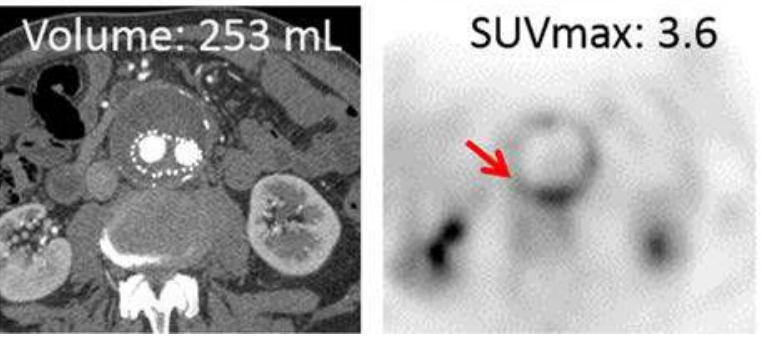

without significant shrinkage of the AAA sac. Aortic foci of FDG uptake are identified by red arrows. CT: X-ray computed tomography, PET: positron emission tomography

percentages of the baseline AAA volume. The AAA walls exhibiting lower baseline FDG uptake have previously been shown to have a lower cell density with an overall low number of cells able to entrap FDG, along with a high degradation of extracellular matrix [23, 24]. It may be hypothesized that this tissue degradation is likely to impair the compliance and remodeling capacity of the arterial walls, whereas these

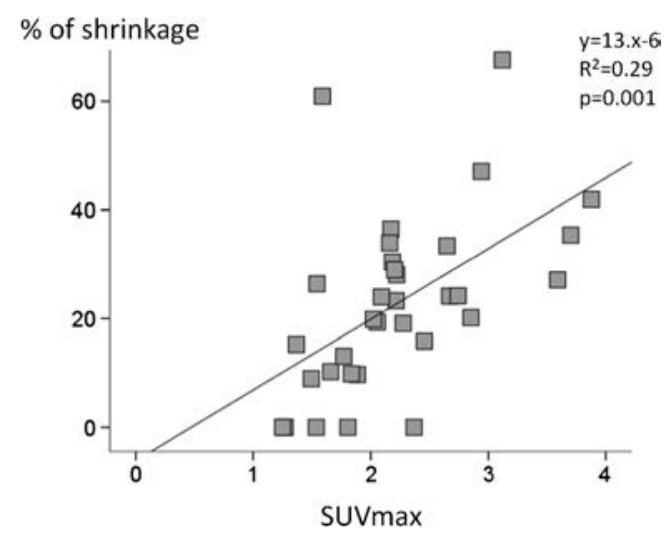

Fig. 4 Presence of an inverse relationship between: 1) the amount of sac shrinkage at 6 months, expressed in $\%$ of total AAA volume at baseline, and 2) FDG activity from AAA (SUVmax) at baseline. The AAA with limited or no shrinkage were the most likely to be those showing a low FDG uptake at baseline. SUVmax: maximal standardized uptake value 
properties are critical for accompanying sac shrinkage after EVAR [2, 25].

Limited sac shrinkage was additionally associated herein with an increase in AAA FDG uptake during the 6-month follow-up period. Moreover, an early and concomitant increase in thrombus volume and FDG uptake was documented as early as at 1 month after EVAR in those AAA showing limited or no shrinkage (see first tercile in Fig. 2). Such increase in FDG uptake has already been shown to accompany the growth phases of medically treated AAA [14], possibly as a result of the recruitment of inflammatory cells at the site of tissue injury [24]. An inflammatory response is far from uncommon early after EVAR, leading to new thrombus onset $[26,27]$, possibly due to the activation of circulating white blood cells, endothelium injury and/or cytokine release from the thrombus during manipulation [26].

Of particular note, no relationship was observed between sac shrinkage and FDG uptake expressed in relation to blood activity (tissue-to-background ratio [14], results not shown), whereas the use of this ratio is currently used for analyzing vascular FDG-PET studies [17]. However, this ratio was previously found to be unrelated to the growth rates of medicallytreated AAA, contrary to SUVmax values $[14,15]$. This is presumably due to the fact that such correction for background activity is not well suited to AAA (i.e. a high proportion of AAA walls are separated from blood by a thrombus layer and their activity are likely to be poorly influenced by blood FDG levels).

\section{Study limitations}

This sequential pilot PET study suffers from a limited sample size, hence the potential reason why certain factors, known to interfere with sac shrinkage, were found not to be significant predictors herein, including statin therapy, active smoking, diabetes, thrombus volume, as well as endoleak and the EVAR system [2, 4, 7, 9-13]. Additionally, endoleaks were no longer correlated with any FDG-PET parameter (results not shown). However, only type 2 endoleaks, thus with limited impact on the shrinkage process [2], were documented in the present study.

It should also be noted that most of our patients ( $82 \%$ ) were treated with the Zenith EVAR device, which may provide better early sac shrinkage than other third-generation stent grafts [4]. However, the extent to which the choice of other systems would have impacted our results remains to be determined.

Because of the limited spatial resolution of the PET images, it is difficult to ensure that the areas of increased FDG uptake were systematically intra-parietal, thereby constituting a further limitation.

An additional limitation is the multicentric nature of this study with the use of different PET/CT systems. It is likely that this variability may be a source of a further imprecisions even if the reconstructed voxel sizes were comparable between the various PET cameras.

Lastly, the impact of these FDG-PET results on clinical endpoints, such as mortality and subsequent rupture and reintervention rates, remains to be fully investigated.

\section{Conclusion}

This study shows that a low pre-EVAR FDG uptake and increased AAA FDG uptake at 6 months are associated with reduced sac shrinkage. This sequential FDG-PET pattern is similar to that already shown to accompany growth phases of medically-treated AAA.

Acknowledgments The authors thank the networks FCRIN INI-CRCT (Cardiovascular and Renal Clinical Trialists) and Cardiovascular Inserm Clinical Investigation Centers-CIC (Nancy CIC-P, Hôpital Européen Georges Pompidou AP-HP, Paris CIC-P, Lille CIC, Nantes CIC, Rouen CIC, Bichat CIC). The authors also thank M. Pierre Pothier for the editing of the manuscript and Henri Boutley who participated to the quantitative analysis of the PET images.

Funding sources The study was sponsored by Nancy CHRU and supported by a DGOS-Inserm "ANR Translationnelle".

\section{Compliance with ethical standards}

Conflict of interest The authors declare that they have no conflict of interest.

Ethical approval All procedures performed in studies involving human participants were in accordance with the ethical standards of the institutional and/or national research committee and with the 1964 Helsinki declaration and its later amendments or comparable ethical standards.

Informed consent Informed consent was obtained from all individual participants included in the study.

\section{References}

1. Ellozy SH, Carroccio A, Lookstein RA, Jacobs TS, Addis MD, Teodorescu VJ, et al. Abdominal aortic aneurysm sac shrinkage after endovascular aneurysm repair: correlation with chronic sac pressure measurement. J Vasc Surg. 2006;43:2-7.

2. Georgakarakos E, Georgiadis GS, Ioannou CV, Kapoulas KC, Trellopoulos G, Lazarides M. Aneurysm sac shrinkage after endovascular treatment of the aorta: beyond sac pressure and endoleaks. Vasc Med. 2012;17:168-73.

3. Patel R, Sweeting MJ, Powell JT. Greenhalgh RM; EVAR trial investigators. Endovascular versus open repair of abdominal aortic aneurysm in 15-years' follow-up of the UK endovascular aneurysm repair trial 1 (EVAR trial 1): a randomised controlled trial. Lancet. 2016;388:2366-74.

4. Bastos Gonçalves F, Baderkhan H, Verhagen HJ, Wanhainen A, Björck M, Stolker RJ, et al. Early sac shrinkage predicts a low risk of late complications after endovascular aortic aneurysm repair. Br J Surg. 2014;101:802-10. 
5. Bisdas T, Weiss K, Eisenack M, Austermann M, Torsello G, Donas KP. Durability of the Endurant stent graft in patients undergoing endovascular abdominal aortic aneurysm repair. J Vasc Surg. 2014;60:1125-31.

6. Houbballah R, Majewski M, Becquemin JP. Significant sac retraction after endovascular aneurysm repair is a robust indicator of durable treatment success. J Vasc Surg. 2010;52:878-83.

7. Lalys F, Daoudal A, Gindre J, Göksu C, Lucas A, Kaladji A. Influencing factors of sac shrinkage after endovascular aneurysm repair. J Vasc Surg. 2017;65:1830-8.

8. Lee JT, Aziz IN, Lee JT, Haukoos JS, Donayre CE, Walot I, et al. Volume regression of abdominal aortic aneurysms and its relation to successful endoluminal exclusion. J Vasc Surg. 2003;38:1254-63.

9. Dias NV, Ivancev K, Malina M, Resch T, Lindblad B, Sonesson B. Intra-aneurysm sac pressure measurements after endovascular aneurysm repair: differences between shrinking, unchanged, and expanding aneurysms with and without endoleaks. J Vasc Surg. 2004;39:1229-35.

10. Sadek M, Dexter DJ, Rockman CB, Hoang H, Mussa FF, Cayne NS, et al. Preoperative relative abdominal aortic aneurysm thrombus burden predicts endoleak and sac enlargement after endovascular anerysm repair. Ann Vasc Surg. 2013;27:1036-41.

11. Yeung JJ, Hernandez-Boussard TM, Song TK, Dalman RL, Lee JT. Preoperative thrombus volume predicts sac regression after endovascular aneurysm repair. J Endovasc Ther. 2009;16:380-8.

12. Fujimura N, Obara H, Matsubara K, Sekimoto Y, Harada H, Inoue $\mathrm{M}$, et al. Comparison of early sac shrinkage with third-generation stent grafts for endovascular aneurysm repair. J Vasc Interv Radiol. 2016;27:1604-12.

13. Sternbergh WC, Conners MS, Tonnessen BH, Carter G, Money SR. Aortic aneurysm sac shrinkage after endovascular repair is devicedependent: a comparison of zenith and AneuRx endografts. Ann Vasc Surg. 2003;17:49-53.

14. Morel O, Mandry D, Micard E, Kauffmann C, Lamiral Z, Verger A, et al. Evidence of cyclic changes in the metabolism of abdominal aortic aneurysms during growth phases: ${ }^{18} \mathrm{~F}-\mathrm{FDG}$ PET sequential observational study. J Nucl Med. 2015;56:1030-5.

15. Kotze CW, Groves AM, Menezes LJ, Harvey R, Endozo R, Kayani IA, et al. What is the relationship between ${ }^{18} \mathrm{~F}$-FDG aortic aneurysm uptake on PET/CT and future growth rate? Eur J Nucl Med Mol Imaging. 2011;38:1493-9.

16. Rudd JH, Coughlin PA, Groves AM. Predicting aortic aneurysm expansion by PET. J Nucl Med. 2015;56:971-3.

17. Rudd JH, Myers KS, Bansilal S, Machac J, Pinto CA, Tong C, et al. Atherosclerosis inflammation imaging with 18F-FDG PET: carotid, iliac, and femoral uptake reproducibility, quantification methods, and recommendations. J Nucl Med. 2008;49:871-8.
18. Mandry D, Tatopoulos A, Chevalier-Mathias E, Lemarié J, Bollaert PE, Roch V, et al. (18)F-fluorodeoxyglucose positron emission tomography combined with whole-body computed tomographic angiography in critically ill patients with suspected severe sepsis with no definite diagnosis. Eur J Nucl Med Mol Imaging. 2014;41: 1924-30.

19. Grandpierre S, Desandes E, Meneroux B, Djaballah W, Mandry D, Netter F, et al. Arterial foci of F-18 fluorodeoxyglucose are associated with an enhanced risk of subsequent ischemic stroke in cancer patients: a case-control pilot study. Clin Nucl Med. 2011;36:85-90.

20. Kauffmann C, Tang A, Therasse E, Giroux MF, Elkouri S, Melanson P, et al. Measurements and detection of abdominal aortic aneurysm growth: accuracy and reproducibility of a segmentation software. Eur J Radiol. 2012;81:1688-94.

21. Morin-Roy F, Kauffmann C, Tang A, Hadjadj S, Thomas O, Piché $\mathrm{N}$, et al. Impact of contrast injection and stent-graft implantation on reproducibility of volume measurements in semiautomated segmentation of abdominal aortic aneurysm on computed tomography. Eur Radiol. 2014;24:1594-601.

22. Van Keulen JW, van Prehn J, Prokop M, Moll FL, van Herwaarden JA. Potential value of aneurysm sac volume measurements in addition to diameter measurements after endovascular aneurysm repair. J Endovasc Ther. 2009;16:506-13.

23. Marini C, Morbelli S, Armonino R, Spinella G, Riondato M, Massollo M, et al. Direct relationship between cell density and FDG uptake in asymptomatic aortic aneurysm close to surgical threshold: an in vivo and in vitro study. Eur J Nucl Med Mol Imaging. 2012;39:91-101.

24. Courtois A, Nusgens BV, Hustinx R, Namur G, Gomez P, Somja J, et al. ${ }^{18}$ F-FDG uptake assessed by PET/CT in abdominal aortic aneurysms is associated with cellular and molecular alterations prefacing wall deterioration and rupture. J Nucl Med. 2013;54: 1740-7.

25. Long A, Rouet L, Vitry F, Albertini JN, Marcus C, Clement C. Compliance of abdominal aortic aneurysms before and after stenting with tissue Doppler imaging: evolution during follow-up and correlation with aneurysm diameter. Ann Vasc Surg. 2009;23: $49-59$.

26. Arnaoutoglou E, Kouvelos G, Koutsoumpelis A, Patelis N, Lazaris A, Matsagkas M. An update on the inflammatory response after endovascular repair for abdominal aortic aneurysm. Mediat Inflamm. 2015;2015:945035.

27. Kakisis JD, Moulakakis KG, Antonopoulos CN, Mylonas SN, Giannakopoulos TG, Sfyroeras GS, et al. Volume of new-onset thrombus is associated with the development of postimplantation syndrome after endovascular aneurysm repair. J Vasc Surg. 2014;60:1140-5. 